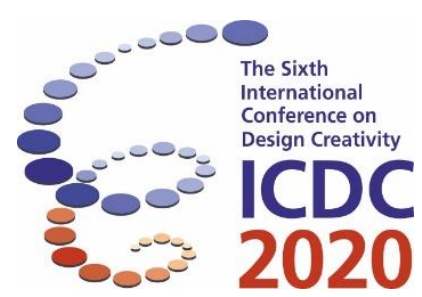

\title{
Facilitating design for the unknown: An inclusive innovation design journey with a San community in the Kalahari Desert
}

\author{
Heike Winschiers-Theophilus ${ }^{1}$, Veera Virmasalo ${ }^{2}$, Marly M. Samuel ${ }^{1}$, \\ Brit Stichel $^{3}$, and Helena E. Afrikaner ${ }^{1}$ \\ ${ }^{1}$ Department of Computer Science, Namibia University of Science and Technology, \\ Windhoek, Namibia \\ ${ }^{2}$ Department of Culture and Society, Malmö University, Malmö, Sweden \\ ${ }^{3}$ Department of Information and Communication, Flensburg University of Applied Sciences, \\ Flensburg, Germany
}

\begin{abstract}
This paper presents a 2-year collaboration that explored how we as design researchers may support an isolated and marginalised indigenous San community to innovate technology products for affluent consumer markets on the other side of the world. The goal was to design a product that brings income to the community, builds skills while facilitating a creative design of self-expression.

Community-based co-design was integrated into an inclusive innovation approach. The resulting product was a series of fridge magnet souvenirs using augmented reality technology. As the community was designing not only for a technology but also for an audience unknown to them, we conceptualised the unknown within the process. Our role extended to facilitating negotiations between commercial goals and communities' creative self-expression. We present the appropriated community-based co-design process and reflect on how our facilitation of the unknown affected the process, the creativity and the self-expression by the San participants.
\end{abstract}

Keywords: inclusive innovation, community-based co-design, San, Namibia

\section{Introduction}

This paper discusses experiences from a 2-year research collaboration with an isolated San community, "the D community", in Namibia. The San are indigenous people of Southern Africa, and their socioeconomic position has been marginalised for generations. They mostly live in small communities scattered around the region, each with their own distinct language. In Namibia, the San represent approximately one percent of the national population. They are faced by lack of access to resources such as land and education, and with limited political representation in government structures. (Suzman, 2001.) Our collaboration with the D community entails multiple projects implemented simultaneously. All have the same underlying double rationale, firstly to contribute to scholarship on participatory community technology design and secondly to support the community to create community-defined well-being in the community. The project discussed in this paper aimed to facilitate the design of an innovative technology product that would bring income to the community. As such, the paper describes a case study in inclusive innovation - albeit differing from the mainstream of inclusive innovation. We want to demonstrate that no matter how marginalised a community is perceived to be, they are able to 
contribute to the innovation of technology products not only for other marginalised people but for affluent consumer markets, too - and this can be done in a way that also contributes to skills development and empowerment through self-expression. In the following sections, we briefly frame our work in the context of innovation and community-based co-design and outline some key theoretical points relevant to the challenges that may emerge when isolated marginalised communities design for commercial gain. We then introduce the design context and participants of this particular project before proceeding to our main contribution, which is a detailed description of the design process, the methodology that was developed and applied. Finally, we present some early stage reflections on our learnings from this project.

\section{Theoretical framing}

\subsection{Commercialised innovation for the unknown through community-based co-design}

"Inclusive innovation" in a development context typically refers to processes where products and services are innovated for the marginalised - sometimes with marginalised users, but often entirely without their involvement. Such processes aim to develop new, improved or more affordable solutions for problems that marginalised communities and individuals are facing in their daily lives. (Heeks et al., 2013.) In line with Chataway and colleagues (2013), we suggest that innovation processes are an effective remedy for exclusion only if the process itself is inclusive of all social groups. Moreover, we hold that the participation of the marginalised should not be restricted to designs for other marginalised people. Instead, marginalised communities have a lot to give and gain from innovating for affluent markets. For this potential to realise, inclusive innovation processes with isolated communities require effective facilitation. A suitable existing approach is community-based co-design (CBCD). Based on the philosophy of participatory design, CBCD emphasises the engagement of communities at all stages of design processes (Blake et al., 2011; Kapuire et al., 2015). This includes decision-making regarding choices of methods, outcomes, timelines and individual involvements. Ideally, CBCD is based on a long-term cooperation (Kapuire et al., 2015) and empowers the community with education and skills (Van Zyl \& Vannini, 2013), often engendering well-being through generating avenues for selfexpression (Sabiescu, 2015). As CBCD typically centres on users known or similar to the community and does not involve commercialisation, our experience suggests that at least three additional factors must be taken into consideration in processes that use this approach to facilitate innovation for commercial goals. 1) Fair and sustainable distribution of profit and other gains must be discussed from the beginning (Marais \& Botes, 2007). 2) The need to market inclusive innovation products for distant clients may be in conflict with the community's deeper needs for self-expression and conflicts need to be recognized and negotiated. 3) To be able to have agency in such negotiation, the community needs to form a picture of their potential clients "out there", and this becomes an important task for the facilitators. Below, we discuss points 2 and 3 with reference to existing literature and illustrate the complexity of the task with the example of the San.

\subsection{Potential conflicts between self-expression, empowerment, and marketing}

The marginalised position of many indigenous groups is in part attributable to their lack of selfexpression. They are unable to determine how others see them, as perceptions are already dominated by narratives and stereotypes defined by the more powerful groups (Stichel et al. 2019). These narratives and stereotypes also affect the self-perception of the marginalised people themselves (Sabiescu, 2015). Through community-designed media, marginalised communities can develop and distribute counternarratives, creative self-expressions, which empower them and may change others' perceptions of them in the long run (Martín-Barbero, 1993). Innovation aiming for commercial gain, however, is dependent on what clients want to buy and this might be different from what the community wants to express. In the case of community-designed products the client often wants to buy the experience of "doing good" (White et al., 2012). Research suggests that to effectively market do-gooding to overseas customers, it pays off to portray communities as relatable individuals whose worries and joys seem familiar to the target market, who are working hard to help themselves but need a little additional support in order to start making headway. This is in contrast with earlier marketing tendencies to portray marginalised 
people as helpless victims. (Seu \& Orgad, 2017.) On the other hand, critiques have also pointed out that the new agentic marketing trend is potentially damaging for struggles against inequality. Warm and relatable "curated stories" of active individuals, obscure structural oppression and the need for confrontational social movements by suggesting that poverty is an issue at the individual level that can be fixed by cultivating entrepreneurial, upwardly mobile subjects (Fernandez, 2017). The risk for exaggerating such quick fix potential may be particularly prominent in the case of marketing and advocacy for technology innovation (Scott-Smith, 2016).

\subsection{The San - stereotypes, self-perceptions and self-expressions}

Stereotypes concerning the San are rife. In Namibia, the San are often seen as alcohol-loving child-like beings who are incapable of keeping a job or otherwise sustaining themselves (Suzman, 2001). There is also a tendency to blame the government for not doing enough for the San (Stichel et al., 2019) and this, too, frames the San as inherently dependent on others to help them. More positive Namibian stereotypes of the San include "technically gifted", "artistic", "clever", "skilled hunters" and "powerful healers" (Suzman, 2001). Internationally, the image of the San is that of hunter-gatherers living in harmony with each other and the nature (Francis \& Francis, 2010), and this view is also gaining ground in urban Namibia (Stichel et al., 2019). Both the domestic and international narratives direct attention to the loss of the hunter-gatherer existence as the major cause for marginalisation, and this partially obscures the role played by current socio-economic discrimination (Francis \& Francis, ibid.).

A group of Namibian San authors (Fernando et al 2018) has stated that the San often grow up assuming the position of a marginalised victim or someone who must constantly fight the stereotypes. The marginalised self-perception was evident in the co-design process, which Stichel and colleagues (2019, see also Stichel, 2018) run with the D community concurrently with the magnets project. Stichel's project created a video dialogue between the community and Namibia's other ethnic groups regarding the representations of the San. The project had no commercial goals, so contributions were not constrained with concerns of what would "sell". Asked what they wanted to tell the outsiders about themselves, the San participants' first choice was to portray the discrimination and the difficulties the community was facing. Only when prompted for thinking about positive self-representations, did they do so and identified the tradition of herbal medicine as something positive to be shared. The community focus on the negative was evident throughout the project, although praise from outsiders for traditional San dances did evoke happiness and pride and, in the end, the community concluded that the project had reconnected them with their traditions. Another result from the project demonstrates that even when the distant audiences were within the same country, the community could not anticipate audience reactions to their videos.

Yet other research with urban San youth in Namibia suggests that while the discrimination faced by the San is real, the self-perception as marginalised might not be all-encompassing. Cabrero and colleagues (2016) noticed in a workshop on user-created personas that San participants highlighted their marginalised position when they worked together with majority ethnic groups. When the San worked among themselves, marginalisation themes did not emerge. The portrayal of self as a victim might therefore be a resource the San use when making claims on others, despite recurring experiences of failing to achieve desired results. In the words of one D community member to Stichel $(2019$, p. 39), "we are left out and even if we complain or something nothing is happening, and it really pains." Contemporary anthropologists of Southern Africa have pointed out that such claims are often made by the marginalized on fellow citizens and visiting tourists and academics as well as on governments. While they may sound like helpless cries of dependency, and while they in part may contribute to further marginalization, they are also a form of agency. Quite simply, constantly reminding others about one's terrible situation is a viable survival strategy in societies where humanity is traditionally seen as interdependence, not independence, and where getting help from others is central for survival as proper employment is scarce and government-led social protection systems are weak. (Durham, 1995; Ferguson, 2013.) 


\section{Design context}

\subsection{The Tech Hub and its research team}

The research collaboration was driven by the Tech Innovation Hub that was newly established at a local university. The tech hub team in the collaboration comprised six people. Two were computer scientists specialised in community-based co-design (CBCD), one a community development practitioner, one a business specialist, and two media and communication researchers and practitioners. Three team members were Namibian, including one San, and three originated from countries where the target market of the products were situated. The collaboration run parallel with research and training on noncommercial digital self-representation with the D community (Stichel et al., 2019). The team also bought professional services in translation and video production as needs arose. The first author of this paper, who is the tech hub lead, has a long experience in implementing CBCD projects with her university and various indigenous communities in Namibia (Winschiers-Theophilus et al., 2017). These collaborations have resulted in numerous prototypes for innovative tech products, none of which have reached to the market due to financial and structural constrains at the university. At the same time, many of the participating communities have expressed a hope that the collaborations would - in addition to other tangible and intangible results - open avenues for livelihoods. The aim of this new research collaboration was therefore precisely to develop a product that would sell in an affluent consumer market. The process could then be used as a proof of concept, demonstrating that it is possible to use CBCD to include marginalised indigenous communities in the design of products that are selling in high-end markets.

\subsection{The augmented reality magnet project}

The idea of augmented reality (AR) fridge magnets as a potentially viable product targeting the souvenir market came from the first author. It was approved by the urban San group at the first stage of the collaboration. An AR fridge magnet is a flat magnet covered with an image which, when scanned by a mobile phone, initiates augmented content on the phone. An AR design process therefore entails producing suitable images for the magnets and developing the augmented content, in this case videos, which again require conceptualisation, scripting, acting, videography and editing. Other production tasks included the compilation of the selected material into a mobile AR application. The innovation process also entails prototyping and market research before the final product design phase, as well as the development of marketing materials in preparation of release to the market.

This paper captures the magnet project at a stage where the collaboration has been on-going for 2 years. A set of nine magnets has been released to the market, a corresponding app is published in the Play Store (ictechhub.com/buy-augmented-reality-souvenirs/), marketing materials have been developed and published online (ibid.) and a small number of magnets have been sold in various events, but the actual marketing work is yet to start. Although the magnet project as such has largely come to completion, collaboration with the community continues with other projects. These include academic research as well as support for the community to develop their own income-generating projects and raise funding for those projects through a crowdfunding platform developed and maintained by the tech hub.

\subsection{The San Participants}

The community collaborators were the rural D community, already referred to, and a group of San youth studying in the capital. The urban youth were invited to participate by the tech hub member who identifies as San herself. The D community was approached based on a recommendation from a bilateral development organisation that works with several San communities. The urban collaborators were approximately 10 students, some of whom were also small-scale entrepreneurs. They participated in the prototyping and market research, while the final magnets with the associated video content were cocreated with the D community, situated 8 hours from the capital, in the fringes of the Kalahari Desert. $\mathrm{D}$ is regarded as one of the most neglected communities in Namibia. It has approximately 200 inhabitants, of which approximately 30 participated actively in the co-creation. The community has no 
infrastructure such as electricity, sanitation or proper cellular coverage. The only road leading to $\mathrm{D}$ is a bumpy sand track only accessible with a $4 \times 4$ vehicle. The community lives in simple houses made out of zinc sheets, wooden sticks and blankets. The vegetation is scarce, permitting minimal farming activity. The only jobs in the vicinity are with poor working conditions and minimal pay. The community has nearly no access to information nor is able to make itself known to outsiders by producing its own media. Many community members do visit the closest town several times a year, but few outsiders ever visit the community.

\section{Methodology as results - the community-based co-design process}

This section gives a detailed description of the community-based co-design (CBDC) process that was developed to design the AR fridge magnets. As such, the section presents both the methodology and the results of the research. A general description of the CBCD approach is given in section 2.1.

\subsection{Prototyping with San students in the capital}

Prototyping was conducted through three workshops with the urban San youth. In the first, different media for image designs were explored. Ostrich eggshells, a traditional San medium, had to be abandoned as they did not result in sufficient contrast for AR technology. The group decided that drawings and photographs should be used instead. Workshop two exposed participants to AR and its applications in bracelets, postcards, children's books, etc. After this experience, participants ideated a set of AR magnets in workshop three. Their ideas for content included, for example, two traditional games popular with San children. One entailed tying a bug (a largish flying insect) to a string and flying it like a kite until it was fried and eaten at the end of the day. In another, children dared each other to snitch an ostrich egg without getting caught by the ostrich. The participants enacted their ideas for videos, which were filmed at the workshop location.

\subsection{Surveying the tourism market with the San students in the capital}

For conducting a market survey, a dedicated game was developed by the tech hub and the urban San youth. The game, based on the idea of the board game Lifestyle, involved presenting images from the prototyping session and asking questions like, "which magnet would you choose for your niece back home?" The youth played the game together with foreign tourists for 2 hours at a popular backpacker's hostel in Windhoek. The gaming session was followed by a mixed group design session where the youth designed magnet concepts together with the tourists. Results from gaming and group design sessions were collated for the market survey, which also entailed conventional surveying based on questionnaires as well as semi-structured interviews with tourists in town. The survey was conducted by the youth with guidance from the tech hub. The market survey concluded that foreigners and Namibians alike are interested in buying souvenirs with nostalgic representations of San traditions, such as dances or hunting techniques. A number of sample images were then selected to inform the next phase. These did not include the bug game, as it could be anticipated to evoke mixed reactions in the target market.

\subsection{Co-design and content production with rural San community in the Kalahari}

The final magnet images and the associated video content were co-created with the D community in the Kalahari. The tech hub team made two one-week visits to community at this stage. First, we explained the idea in a community meeting. After an agreement on collaboration was achieved, the magnet prototypes were demonstrated, results of the market survey presented and discussed in groups. The community was taken through a process where they developed the prototypes further. Participants selected images they thought most relatable. They drew their own images inspired by the prototypes and shared associated personal and traditional stories, which were captured on video. The community then decided which themes should be enacted into videos for the magnets and which community members were the best actors for specific themes. All identified themes were traditional practices, which the community no longer practices in their daily life. A variety of scenes were then shot with professionals operating the video camera and drone. The participants took part in recoding video and audio and assisted in directing. They learned how to make videos, organize a film set and brainstorm 
about media ideas. The second visit started with the screening of two magnet videos that had been edited in Windhoek based on the material filmed in the first visit. Feedback was sought from the community, as well as ideas for the remaining videos, which were to be ideated, acted and filmed in this visit. Accompanying images also had to be produced. A San graphic designer based in Windhoek joined the team and worked with the community to identify the drawings and photographs most suitable for the AR technology. This resulted in nine different magnets. The augmented video content depicted hunting with bow and arrow, uses of a traditional herbal perfume and a particular tree, a healing session, and a traditional dance.

A separate session was held with the entire community to determine business models and payments. After long discussions and much opposing views within the community, it was agreed that the individuals contributing their time and effort to the production should be compensated immediately and individually in relation to their efforts. Further, the profit of the magnet sales should be deposited on a community account and only utilised once it reaches a certain amount needed to purchase items the community had agreed on. Legitimate purchases included building material and transport services beneficial to the entire community. The meeting also agreed on how much tech hub and other visiting researchers should pay for the community for camping, cooking and other services.

\subsection{Marketing material design with rural community in the Kalahari}

Once the magnets were ready for the market, the research team returned to the community for two days to conduct a workshop, which would co-design marketing materials and develop skills in marketing and possible future dealings with media people. Nine community members participated. They were first asked, through a peer interview and photography exercise, what they would like their faraway customers, "like the facilitator's mother", to know about them. This exercise produced narratives about what the community was lacking and wanted to be provided to them, including proper houses, road, car, land ownership and their own animals. We then told the participants that research suggests people are likely to react negatively if all they hear is misery, complaints and demands. Could they tell something good about the life in the community, including the joys of the individuals who appear in the magnet images and videos? While morsels of additional information came out, the participants repeatedly brought the conversation back to their misery and their longing for the past that was lost. The issue did not seem to be that they did not feel comfortable with sharing intimate thoughts. Instead, they could not imagine why mundane things about an individual would interest a stranger. The interviews and the follow-up discussion was then collated in community member profiles and their potential uses in marketing were discussed. Each profiled community member listened to their portrait in private and decided if they approved the use of it and whether they wanted to change something. The participants listened to the reading of their portraits with solemnity and seemed to be genuinely pleased to be "talking" to the outside world on behalf of the community. The final profiles were only slightly altered from the first narratives. The two older community members' longing for the past was amended by their thoughts on how income-generating projects may make a difference in the future. The young men's struggles to find work, on the other hand, were complemented with their values that stemmed from the community and its cultural traditions, while the craft-making women shared their wishes for their young children.

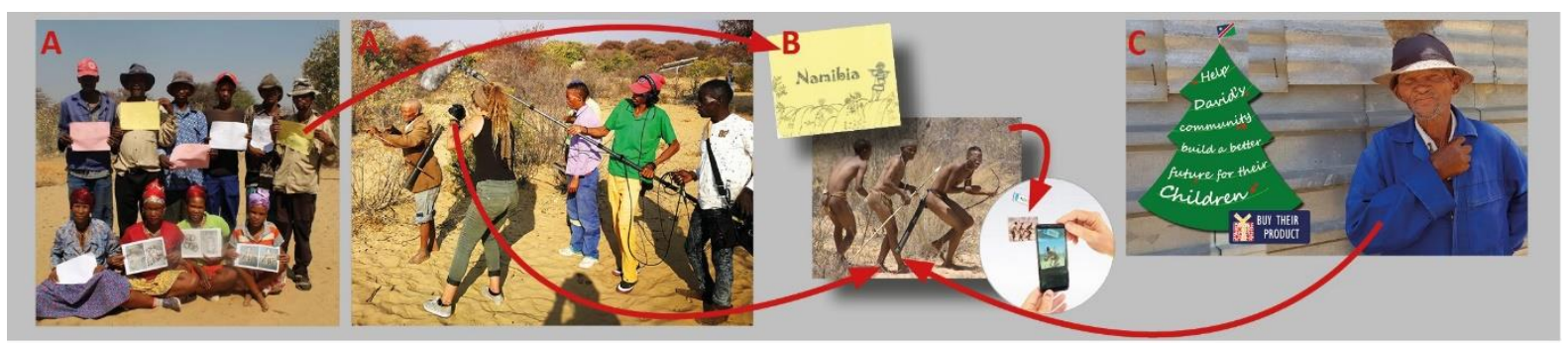

Figure 1. Collaborative production of magnet imagery and augmented content with the Donkerbos community (A), two of the resulting augmented reality fridge magnets in use (B), co-designed social media marketing banner targeting overseas Christmas sales (C). 


\section{Discussion and Conclusion}

Above, we demonstrate that no matter how marginalized a community is perceived to be, they are able to contribute to the creative design of tech innovations not only for other marginalized people but for affluent consumer market, too. The two San communities participated in the design of the AR fridge magnets from the onset and their contributions are the defining feature of the final product, which is already selling in overseas markets. The community applied their creativity to a technology, which was previously unknown to them, with results that appeal to audiences who they originally knew very little about. The San creativity materialized in images and videos in which they expressed their longing for the past. The creative process ignited a sense of pride and reconnection with traditions. On the other hand, it also produced critical narratives of the present.

The community gain is not restricted to this single project. They have established a long-term partnership with us, which is based on mutual learning and is beneficial to both parties. The community has often expressed deep gratitude for us "coming back". To them, our repeated visits and our emphasis on learning from them, testifies to our humanness and tells them we have respect for them. Such relationship is a relationship of connectedness rather than of dependency. It allows community members to expand their boundaries in exploring "creativity at the extremes" as they have, through the collaboration with us, opportunities to take part in designing with emerging technologies such as AR and virtual reality.

The process also contributed to skills development. While the urban youth learned about market surveys, the rural community is now well-prepared to negotiate financial and other terms of their involvement in any production and to learn more in the future. They know what kinds of tasks, technologies and skills are involved in media production, and have tried their hand at all these aspects. If they had the possibility to use quality mobile phone cameras with microphones, they would be able to produce raw material that would be shareable on social media and acceptable for broadcasting by most mainstream media outlets, although not suited for the production of sleek adverts targeting affluent consumer markets. Most relevantly to this paper, both communities formed a picture of their unknown clients "out there". This understanding is useful in the future, if they want to employ their creativity to generate livelihoods or to express themselves with other intentions to a similar audience. To achieve this learning, we as the facilitators of design for the unknown did have to steer the process in ways that would rarely be advisable in community-based co-design processes, which do not have commercial goals and aim for unstifled creativity and self-expression by the participants. For example, the process entailed a market survey, which directed the production themes to the picturesque and exotic past. We also suggested pruning off the kinds of representations of the past that did not fit in the stereotype of harmonious coexistence with the nature, and hence the magnets have no reference to bugs that are played with and then eaten. Neither is the community members' longing for hunting and eating bush meat portrayed as strongly in the marketing materials as it was in the original community narratives. Most critically, when the community wanted to emphasize their misery in the marketing materials, we told them that the audience probably does not want to hear only complaints and demands.

In the context of the self-expression theory, the above can be seen as constraining community agency. However, the issue is more complex. As already stated, the community learned skills that they will be able to use to develop self-expressions for other contexts. They were also not blindly following our suggestions. For example, the final marketing materials retained a good deal of the complaints the community wanted to express. We suggest that it is critical to recognize and discuss the conflicts that potentially arise between marketing needs and the community's deeper needs for self-expression. This is an important part of the learning. For example, as important as self-expression about grievances is for the D community and to their goal to gain recognition and redress, the design process also planted seeds for reflections on whether self-expression as a victim is also contributing to the kind of selfreproduction of victimhood, which prevents the community, its individuals - and ultimately also outsiders - from seeing community capabilities. Another complex issue is the emphasis on cultural traditions that are no longer practiced. On the one hand, this reinforces the stereotype of the San as frozen in time. On the other hand, the magnets project confirmed Stichel and colleagues' (2019) findings that reconnection with cultural traditions engendered pride and happiness in the community. Therefore, 
creative self-expression featuring traditions in design was genuine, not just something engineered to attract customers.

\section{Acknowledgements}

We are most grateful to the community members who have participated in this project and enriched us with their creativity. We acknowledge the funding support by the Embassy of Finland, Windhoek for the tech innovation hub and this project in particular.

\section{References}

Blake, E. H., Tucker, W. D., Glaser, M., \& Freudenthal, A. (2011). Deaf telephony: community-based co-design. Interaction design: beyond human-computer interaction, 3, 412-413.

Cabrero, D. G., Winschiers-Theophilus, H., Abdelnour-Nocera, J., \& Kapuire, G. K. (2016, August). A hermeneutic inquiry into user-created personas in different Namibian locales. In Proceedings of the 14th Participatory Design Conference: Full papers-Volume 1 (pp. 101-110).

Chataway, J., Hanlin, R., \& Kaplinsky, R. (2014). Inclusive Innovation: An Architecture for Policy Development. Innovation and Development, 4 (1): 33-54.

Durham, D. (1995). Soliciting gifts and negotiating agency: the spirit of asking in Botswana. Journal of the Royal Anthropological Institute, 111-128.

Ferguson, J. (2013). Declarations of dependence: labour, personhood, and welfare in southern Africa. Journal of the Royal Anthropological Institute, 19(2), 223-242.

Fernandes, S. (2017). Curated stories: The uses and misuses of storytelling. Oxford University Press.

Fernando, K., Fernandu, T., Kapembe, S., Isay, K., \& Hoffeni, J. (2018). A Contemporary Expression of the Namibian San Communities' Past and Present Sufferings Staged as an Interactive Digital Life Performance. In Digitisation of Culture: Namibian and International Perspectives (pp. 205-221). Springer, Singapore.

Francis, M. \& Francis, S. 2010. Representation and misrepresentation: San regional advocacy and the global imagery. Critical Arts. July 2010.

Heeks, R., Amalia, M., Kintu, R., \& Shah, N. (2013). Inclusive innovation: definition, conceptualisation and future research priorities. development informatics working paper, (53).

Kapuire, G. K., Winschiers-Theophilus, H., \& Blake, E. (2015). An insider perspective on community gains: A subjective account of a Namibian rural communities' perception of a long-term participatory design project. International Journal of Human-Computer Studies, 74, 124-143.

Marais, L. \& Botes, L. (2007). Income generation, local economic development and community development: paying the price for lacking business skills? Community Development Journal, 42(3), 379-395.

Martín-Barbero, J. (1993). Communication, Culture \& Hegemony: From the media to mediations. London: Sage Sabiescu, A. (2015). Narratives and counter-narratives in the representation of The Other. The case of the Romani ethnic minority. Informing Science.

Scott-Smith, T. (2016). Humanitarian neophilia: the 'innovation turn' and its implications. Third World Quarterly, $37(12), 2229-2251$.

Seu, I. B., \& Orgad, S. (2017). Caring in crisis? Humanitarianism, the public and NGOs. Springer.

Stichel, B. (2019). A mediated intermedia conversation around the perception and self-perception of the San. Master Thesis at Hochschule Flensburg. Unpublished.

Stichel, B., Blake, E., Maasz, D., Stanley, C., Winschiers-Theophilus, H., \& Afrikaner, H. (2019, June). Namibian Indigenous Communities Reflecting on Their Own Digital Representations. In Proceedings of the 9th International Conference on Communities \& Technologies-Transforming Communities (pp. 51-59). ACM.

Suzman, J. (2001). Regional Assessment of the Status of the San in Southern Africa. Report No. 4 of 5. Windhoek: Legal Assistance Centre (LAC).

Van Zyl, I., \& Vannini, S. (2013). Participatory re-action: reflecting on a Design-Based Research approach in ICT4D. In Public and private access to ICTs in developing regions. Proceedings of the 7th International Development Informatics Conference, Bangkok, Thailand. 1-19.

White, K., MacDonnell, R., \& Ellard, J. H. (2012). Belief in a just world: Consumer intentions and behaviors toward ethical products. Journal of Marketing, 76(1), 103-118.

Winschiers-Theophilus, H., Zaman, T., \& Stanley, C. (2017). A classification of cultural engagements in community technology design: introducing a transcultural approach. Ai \& Society, 1-17. 